\title{
A Rare Complication of PDA Coil Occlusion: Rapidly Migrating Foreign Body from Femoral Vein to Right Ventricle
}

\author{
Ozgur Daga, Sureyya Talaya, Mehmet Ali Kaygin ${ }^{\mathrm{a}}$, Bilgehan Erkut ${ }^{\mathrm{a}, \mathrm{b}}$
}

\begin{abstract}
We report the case of a 4 year young female patient with a complication of patent ductus arteriosus during coil occlusion. Preoperative radiological scopy on the operating table demonstrated the coil which migrated from iliac vein to right ventricle during short transport time. Patient underwent an emergent aorto- bicaval cannulation with sternotomy and removal of the coil under cardiac arrest via right atriotomy. Consequently, we strongly advocate that these percutaneous techniques can only be performed in circumstances with backup possibilities by cardiovascular surgeons.
\end{abstract}

Keywords: PDA; Coil occlusion; Complication; Surgery

\section{Introduction}

First successful surgical patent ductus arteriozus (PDA) ligation is reported by Dr. Robert E. Gross in 1939 [1]. Left posterolateral thoracotomy is the standard surgical approach in PDA cases for decades after Dr. Gross. Median sternotomy was also administered by a smaller group of surgeons. Over the past several years treatment of PDA considerably evolved through less invasive techniques such as percutaneous transcatheter coil occlusion, video-assisted thoracoscopic occlusion (VATS) and non-surgical indometasin treatments.

Despite the modern days tendency of less invasive and smaller incision therapeutic options, morphology and location of PDA, clinical situation with age and size of the patient and the experience of a clinic determine the technique of choice.

\footnotetext{
Manuscript accepted for publication October 5, 2012

${ }^{a}$ Erzurum Regional Training and Research Hospital, Cardiovascular Surgery Department, Erzurum, Turkey

${ }^{\mathrm{b}}$ Corresponding author: Bilgehan Erkut, Erzurum Region Training and

Research Hospital, Cardiovascular Surgery Department, Erzurum,

Turkey. Email: bilgehanerkut@yahoo.com
}

doi: http://dx.doi.org/10.4021/jmc350w

\section{Case Report}

A 4 year young female patient administered to the pediatric department with a history of dyspnea episodes and frequent attacks of pneumonia. Initial physical examination and transthoracic echocardiography demonstrated a PDA as typically located between left pulmonary artery and aorta. Following the routine clinical evaluation, hematological tests and chest roentgenogram were performed. Attempting pediatrist decided to occlude PDA percutaneously by a coil. Catheterization initiated via artery and venous access with a pediatric anesthesiologist providing sedation. A 4F sheath was placed into the femoral artery and a $5 \mathrm{~F}$ sheath into the femoral vein. Adequate heparin and cefazoline antibiotic prophylaxis were given as the case progresses. Estimation of PDA diameter was obtained by angiography which demonstrated a flow pattern in through the PDA and a moderate diameter less than $2 \mathrm{~mm}$ (Fig. 1). A 5F venous sheath was used in order to deliver the coil and its adjacent guide wire to aortic side. Inadaptability and incompatibility of diameter differences between the sheath and coil adjacent resulted with separation of the coil in femoral vein side. Disconnected part of the coil was observed stable in the femoral vein sheath. At that

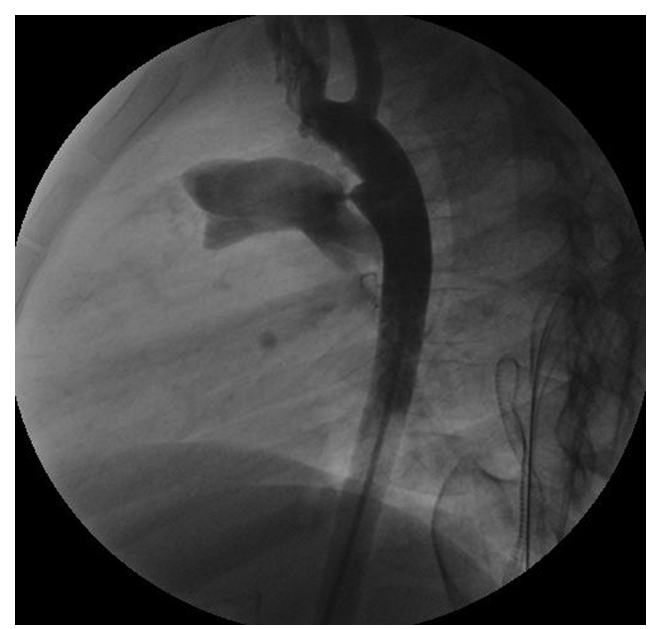

Figure 1. The angiography view of the PDA. 


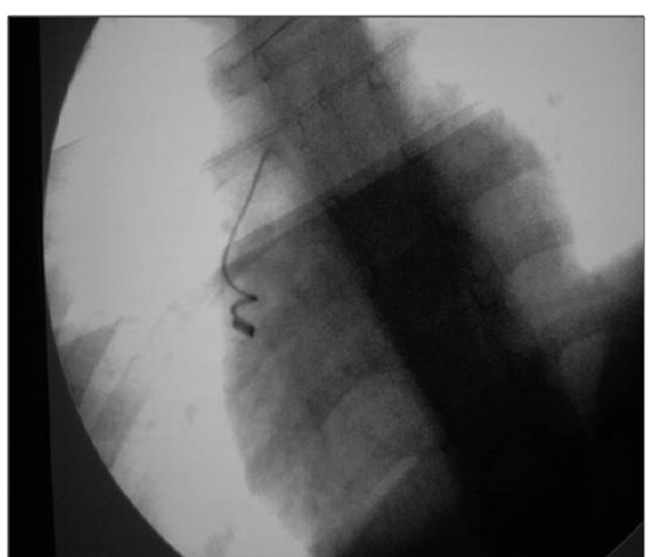

Figure 2. Preoperative on the table scopy of Coil immigration into heart.

phase the catheterization was ended and the child referred for surgery as emergency.

On the operating table, physical examination presented an appearance of respiratory distress with cyanotic limbs. Electrocardiogram revealed sinus tachycardia with a rate of 180 per minute. Routine monitoring included transcutaneous oxygen saturation, arterial blood pressure, and electrocardiogram. Preoperative radiological scopy to determine the definitive localization demonstrated that the coil migrated from iliac vein to right ventricle during short transport time (Fig. 2). Under general anesthesia, a median sternotomy was initially performed. Heparin injection with 350 units $/ \mathrm{kg}$ to reach the activated clotted time for over 400 seconds. Following pericardiectomy, aorto-bicaval cannulation was performed within $28^{\circ} \mathrm{C}$ of body core temperature. Cross clamp was applied to both aorta and pulmonary artery to prevent the continuation of the coil migration more distally through the lungs. Cardiopulmonary bypass was accomplished with centrifugal pump and membrane oxygenator. For myocardial protection, we used three steps of cardioplegia: $500 \mathrm{~mL}$ of normothermic blood cardioplegia, cold cardioplegia in 10 $\mathrm{mL} / \mathrm{kg}$ in $40 \mathrm{mmHg}$ pressure. Neither additive cardioplegia doses were necessary due to the short period of cardiac arrest. After securing the caval blood flow with tightened snears over cannulas, right atriotomy performed. Coil was observed between the tricuspid valve structures and reaching into the right ventricle with its free end. Coil removed gently to avoid any harm on tricuspid valve (Fig. 3). Right atriotomy closed and cardiopulmonary bypass ended. Total cardiopulmonary time was 41 minutes and total cross clamp time was 17 minutes. Heparin antagonization maintained by protamine sulfate. Existing PDA between left pulmonary artery and aorta occluded with transfixion sutures with clear visualization. An epicardial pace-maker was replaced. Following the homeostasis maneuvers, surgical drains replaced and sternum closed. Patient transported to the cardiovascular surgery intensive care unit. The intubation time was 180

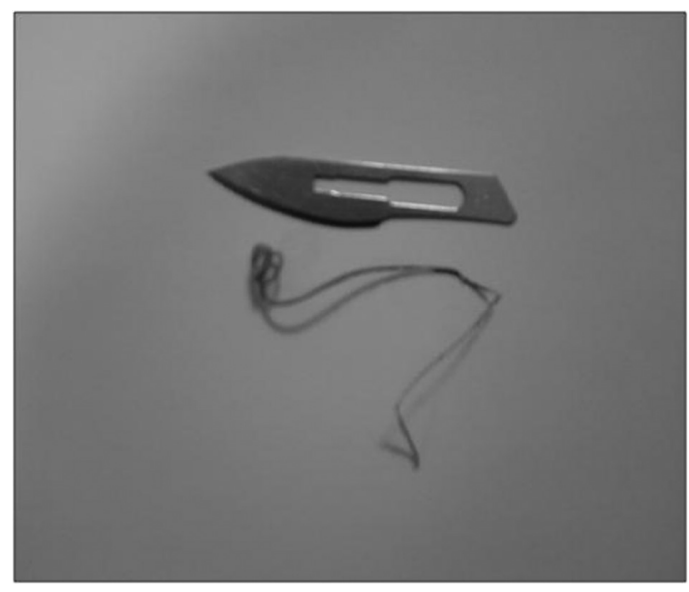

Figure 3. Postoperative picture of removed coil.

minutes and total drainage volume was 210 milliliters. Postoperative transthoracic echocardiograms approved the closure of PDA. The child was discharged at the postoperative day of 8 , uneventfully.

\section{Discussion}

Fetal circulation is maintained by ductus arteriosus allowing most of the blood from the right ventricle to aorta in circumstances of by-passing the immature pulmonary bed. As defined clearly, ductus arteriosus is closed spontaneously by multi-factorial conditions at the early postpartum period in the first 15 hours functionally.

Persistent patency of ductus arteriosus, defined as PDA, is a relatively common congenital cardiac abnormality. Overall incidence differs between $0.02 \%$ and $0.006 \%$ of live births. High altitude births, perinatal asphyxia, lower birth weights, and premature births may elevate the risk of PDA. Clinical presentation differs on age and symptoms depending on the size and natural of the PDA. A left to right flow pattern usually causes with overload of pulmonary vascular resistance. Uncommonly, an adult may present with a murmur which is caused by a small and tolerated PDA.

Morphologically, PDA is mostly located between the left pulmonary artery and proximal part of the descending aorta. Some rare cases may present between the right pulmonary artery and main pulmonary artery. Atypical localizations tend to be concordant to other cardiac and other defects. Mortality rates differ between $0.5 \%$ to $4 \%$ depending on the severity of left to right shunt and age of the case [2]. Different diagnoses may include sinus of valsalva aneurysm, aortopulmonary window, coronary artery fistula and atypical forms of Fallot's Tetralogy.

Treatment of PDA starts with an administration of indometasin or ibuprofen at the early diagnoses [3]. Treatment success of intravenous medications is questionable in some 
cases. Thus, over the last three decades several techniques of catheterization closure are described [4]. Success rates are significantly higher for PDA within a diameter smaller than $2.5 \mathrm{~mm}$. PDA equal or larger than $3 \mathrm{~mm}$ diameter is a subject of other occlusion techniques rather than catheterizations. These risks of catheterization may include embolization of occlusive device, recurrent PDA, central or peripheral arterial or venous vascular injury, stroke, mild to severe lower limb ischemia and deaths. Large ductus treatments include conventional left thoracotomy and VATS. Thoracotomy approach may result with surgical ligation or surgical ligation with division. This technique has a very low mortality and morbidity risk on experienced hands, and has achieved excellent results. VATS is a more difficult technique especially for cases with calcification, bigger ductus with a wider risk of hemorrhage and vascular injury [5]. In the presence of a doubt on VATS or conventional posterolateral thoracotomy, a possibility for median sternotomy with or without cardiopulmonary bypass always remains as a last resort for a cardiovascular surgeon.

In our opinion, percutaneous coil occlusion techniques are cost-effective, safe, effective on recurrence rates and less traumatic for the patient. Small to moderate PDA within 1 to $2 \mathrm{~mm}$ diameters has a chance for percutaneous transcatheter coil occlusion. Larger PDA or insufficient PDA neck morphology requires a surgical approach for the best outcomes. Coil occlusions are also with certain rates of complications, as in our case. Thus, these techniques may only be wisely performed with a possibility of surgical support for complicated cases of emergency.

\section{References}

1. Gross RE, Hubbard JP. Landmark article Feb 25, 1939: Surgical ligation of a patent ductus arteriosus. Report of first successful case. By Robert E. Gross and John P. Hubbard. JAMA. 1984;251(9):1201-1202.

2. Dudell GG, Gersony WM. Patent ductus arteriosus in neonates with severe respiratory disease. J Pediatr. 1984;104(6):915-920.

3. Sekar KC, Corff KE. Treatment of patent ductus arteriosus: indomethacin or ibuprofen? J Perinatol. 2008;28(Suppl 1):S60-62.

4. Cambier PA, Kirby WC, Wortham DC, Moore JW. Percutaneous closure of the small (less than $2.5 \mathrm{~mm}$ ) patent ductus arteriosus using coil embolization. Am J Cardiol. 1992;69(8):815-816.

5. Laborde F, Folliguet T, Batisse A, Dibie A, da-Cruz E, Carbognani D. Video-assisted thoracoscopic surgical interruption: the technique of choice for patent ductus arteriosus. Routine experience in 230 pediatric cases. J Thorac Cardiovasc Surg. 1995;110(6):1681-1684; discussion 1684-1685. 